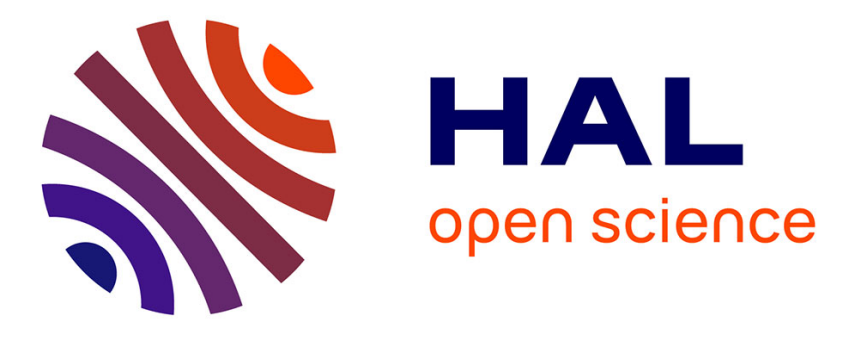

\title{
Dynamic grouping maintenance strategy with time limited opportunities
}

Phuc Do Van, Brissaud Florent, Bouvard Keomany, Anne Barros, Christophe

Bérenguer

\section{- To cite this version:}

Phuc Do Van, Brissaud Florent, Bouvard Keomany, Anne Barros, Christophe Bérenguer. Dynamic grouping maintenance strategy with time limited opportunities. Annual Conference of the European Safety and Reliability Association, ESREL 2011, Sep 2011, Troyes, France. pp.CDROM. hal00695779

\section{HAL Id: hal-00695779 \\ https://hal.science/hal-00695779}

Submitted on 9 May 2012

HAL is a multi-disciplinary open access archive for the deposit and dissemination of scientific research documents, whether they are published or not. The documents may come from teaching and research institutions in France or abroad, or from public or private research centers.
L'archive ouverte pluridisciplinaire HAL, est destinée au dépôt et à la diffusion de documents scientifiques de niveau recherche, publiés ou non, émanant des établissements d'enseignement et de recherche français ou étrangers, des laboratoires publics ou privés. 


\title{
Dynamic grouping maintenance strategy with time limited opportunities
}

\author{
Phuc Do Van, Florent Brissaud, Anne Barros, Christophe Bérenguer \\ Troyes University of Technology, Institut Charles Delaunay \& UMR CNRS STMR, Troyes, France
}

\author{
Keomany Bouvard \\ Volvo Technology
}

\begin{abstract}
This paper presents a dynamic grouping maintenance strategy for multi-component systems with positive economic dependence, which implies that combining maintenance activities is cheaper than performing maintenance on components separately. Preventive maintenance durations and occurrences of maintenance activities within the scheduling horizon are considered. Moreover, in presence of opportunities with limited durations in which some maintenance activities could be executed with reduced maintenance costs, the present paper proposes a new algorithm to optimally update online the grouping maintenance planning. A numerical example of a five components system is finally introduced to illustrate the proposed dynamic grouping maintenance strategy.
\end{abstract}

\section{INTRODUCTION}

Maintenance models of multi-unit systems are concerned with optimal maintenance policies for sets of components that may be stochastically dependent on each other (transition probabilities between components states depend on other components states) and/or economically dependent (the cost of maintenance of a group of components does not equal the total cost of individual maintenance of these components), see (Cho \& Parlar 1991). Only this second kind of dependencies is assumed in the proposed paper. A review of related maintenance models has been notably proposed by (Nicolai \& Dekker 2007). Economic dependence can save maintenance costs in some cases, e.g. due to economies of scale or positive economic dependence which implies that combining maintenance activities is cheaper than performing maintenance on components separately. On the other hand, grouping maintenance may also lead to higher costs, e.g. due to manpower restrictions, or may not be allowed. The present paper considers only a positive economic dependence.

A major challenge of the maintenance optimisation then consists in joining the stochastic processes regarding to the components (time-dependent probabilities of failure) with the combinatorial problems regarding to the grouping of maintenance activities. While a long term or infinite planning horizon can be assumed to solve this problem in case of stable situations, dynamic models have been introduced in order to change the planning rules according to short-term information (e.g. failures and varying deterioration of components), using a rolling (finite) horizon approach (Wildeman, Dekker, \& Smit 1997). This approach is however applicable only when maintenance durations are neglected. From a practical point of view, the system may be stopped during maintenance of its components, maintenance durations should therefore take into account, especially when the system unavailability cost rate is expensive. Moreover, each component is assumed to be preventively maintained only one time within the scheduling interval. This assumption seems to be not relevant since a system may be composed of different components with different life time cycles, maintenance frequencies of components are thus different. For example, engine oil has to change more frequently than driving belt on a heavy vehicle (Bouvard, Artus, Berenguer, \& Cocquempot 2011). The first objective of this paper is to develop the rolling horizon approach by taking into account both the preventive maintenance durations and the occurrences of maintenance operations in the considered scheduling horizon.

In the framework of opportunities maintenance, the downtime of a system is often an opportunity to combine preventive and corrective maintenance. This is especially true for series systems, where a single failure results in a system downtime, see (Radouane, Alaa, \& Djamil 2009). Preventive maintenance at opportunities with restricted duration, which can randomly occur, can be found in (Dekker \& Smeitink 
1991, Dekker 1995). In such papers, the optimal components for each opportunity are chosen according to their important ranking in terms of maintenance costs. The second objective is to extend the developed rolling horizon approach by taking into opportunities with limited durations in which the system is stopped for whatever reason (for example, relying on a production planning). This can help to online update the grouping maintenance planning in presence of opportunities.

This paper is organized as follows. Section 2 is devoted to the description of general assumptions and maintenance policy. Second 3 focuses on the development of the rolling horizon approach. The online update of grouping maintenance planning in presence of opportunities is considered in Section 4. To illustrate the proposed grouping maintenance strategies, a simple numerical example is introduced in Section 5. In addition some numerical results are discussed here. Finally, the last section presents the conclusions drawn from this work.

\section{NOTATION LIST}

$N \quad$ number of components of the system

$i$ index for components/maintenance operations, with $i=1, \ldots$

$\lambda_{i}, \beta_{i}$ scale and shape parameters of component $i$

$S \quad$ set-up cost

$C_{i}^{p} \quad$ preventive maintenance cost of component $i$

$C_{i}^{c} \quad$ corrective maintenance cost of component $i$

$d_{i} \quad$ preventive maintenance duration of component $i$

$C^{d} \quad$ unavailability cost rate of the system

$t_{\text {begin }}, t_{\text {end }}$ beginning and ending dates of the scheduling interval

$x_{i}^{*} \quad$ nominal preventive maintenance periodicity of component $i$

$i^{j} \quad j$ th maintenance occurrence of component $i$, with $i, j=1, \ldots$

$t_{i^{j}} \quad$ tentative execution date of operation $i^{j}$

$t_{i^{j}}^{*} \quad$ executed maintenance date of $i^{j}$

$h_{i}($.) penalty cost function of preventive maintenance of component $i$

$G^{k} \quad k$ th group of maintenance activities in the planning horizon (defined by a set of $i^{j}$ )

$t_{G^{k}} \quad$ beginning maintenance date of $G^{k}$

$Q_{G^{k}}^{*}$ maintenance cost savings of $G^{k}$

$Q_{\sum}^{*}$ total maintenance cost savings within the scheduling interval

$T_{o p} \quad$ date of the opportunity in the planning horizon

$D_{o p}$ maximal duration of the opportunity

\section{PROBLEM FORMULATION}

\subsection{General assumption}

Consider a system consisting of $N$ components in which a preventive/corrective maintenance of one or more components leads to the system being unavailable. We assume failure rate of a component $i(i=$ $1, \ldots, N)$ is described by a Weibull distribution with scale parameter $\lambda_{i}>0$, and shape parameter $\beta_{i}>1$, then

$r_{i}(t)=\frac{\beta_{i}}{\lambda_{i}}\left(\frac{t}{\lambda_{i}}\right)^{\beta_{i}-1}$.

We assume that the deterioration of a component remains unchanged during the maintenance of other ones.

Each component is preventively maintained after a fixed interval to be optimized. After a preventive maintenance action, the maintained component is considered as good as new. The preventive maintenance cost of the component $i$ can be divided in three parts: a setup-cost; a specific component $\operatorname{cost} c_{i}^{p}$; and an unavailability cost. The setup-cost, denoted $S$, represents the logistic cost and can be shared when several maintenance activities are performed in the same maintenance occasion since execution of a group of maintenance activities requires only one set-up. A preventive maintenance of component $i$, so-called activity $i$, leads the system to be unavailable during $d_{i}$ units of time. Hence when a activity $i$ is carried out, an additional cost or unavailability $\operatorname{cost} c_{i}^{d}=d_{i} \cdot C^{d}$ relying on the production lost is incurred $\left(C^{d}\right.$ is the unavailability cost rate of the system). As consequence, if a preventive maintenance of component $i$ is carried out, we have to pay the following preventive cost:

$$
C_{i}^{p}=S+c_{i}^{p}+c_{i}^{d} .
$$

Note well that the unavailability cost can be shared only when maintenance activities are performed simultaneously. We consider however in this paper that all maintenance activities performed in a maintenance occasion (a group) are alternately executed. Therefore, this unavailability cost can not be shared here.

Between two preventive maintenance intervals, if component fails, it is then repaired immediately. A corrective action restores the component involved into a state as good as before (minimal repair policy). Therefore, when a corrective action is carried out for component $i$, it requires a corrective maintenance cost denoted $C_{i}^{c}$. We assume here the repair duration can be neglected and the corrective maintenance setupcost is already taken into account in $C_{i}^{c}$.

\subsection{Grouping maintenance policy}

Based on the preventive/corrective maintenance costs and the reliability parameters of components, it is possible to define for each individual maintenance activity $i$ a nominal periodicity $x_{i}^{*}$ that minimizes the induced long-term average costs (Wildeman, Dekker, \& Smit 1997), assuming that the maintenance activities are separately performed. 
As mentioned above, the grouping of some maintenance activities should reduce the setup-cost. We are therefore interested to construct a grouping maintenance planning in which in each maintenance occasion some maintenance activities to be optimized are carried out. Note well however that when some maintenance activities are performed in the same maintenance occasion, the maintenance cost could be indirectly penalized

- with the reduction of components useful life if the maintenance dates are advanced;

- with the increasing of components failure probability which could imply a system immobilization if the maintenance dates are too late.

In order to find the optimal groups which could balance to minimize the system maintenance cost on the scheduling horizon, the rolling horizon approach introduced recently in (Wildeman, Dekker, \& Smit 1997, Wildeman \& Dekker 1997) will be developed by taking into account the preventive maintenance durations and the occurrences of maintenance operations, see Section 3.

In presence of an opportunity with limited duration in which the system is stopped, the current grouping maintenance planning could be no longer the optimal one. A new grouping maintenance taking into account the opportunity is needed. The later will be discussed in Section 4.

\section{EXTENSION OF ROLLING HORIZON APPROACH}

The developed approach is divided into 4 steps:

- individual optimization;

- tentative planning;

- grouping optimization;

- update and decision.

\subsection{Step 1: Individual optimization}

The objective is to find optimal individual preventive maintenance cycle based on an infinite-horizon maintenance model in which we assume an average use of component $i$ and in which the interactions between components are neglected.

Let $M_{i}(x)$ denote the expected deterioration cost for component $i$, i.e. the expected costs incurred in $x$ time units since the latest execution of activity $i$. According to a minimal repair policy, $M_{i}(x)$ can be written as the following:

$M_{i}(x)=C_{i}^{c} \cdot \int_{0}^{x} r_{i}(y) d y$, where $r_{i}($.$) is the rate of occurrence of failure of com-$ ponent $i$. $\int_{0}^{x} r_{i}(y) d y$ represents the expected failure numbers of component $i$ within interval $[0, x]$. From equations (1) and (3), the expected deterioration for component $i$ can be expressed:

$M_{i}(x)=C_{i}^{c} \cdot\left(\frac{x}{\lambda_{i}}\right)^{\beta_{i}}$.

If component $i$ is preventively maintained at $x$, the expected maintenance cost within interval $[0, x]$ is then:

$\Gamma_{i}(x)=C_{i}^{p}+M_{i}(x)$.

Using the renewal theory, the long-term average cost $\phi_{i}(x)$ when executing the preventive maintenance of component $i$ every $x$ time units amount is:

$\phi_{i}(x)=\frac{\Gamma_{i}(x)}{x}=\frac{C_{i}^{p}+C_{i}^{c} \cdot\left(\frac{x}{\lambda_{i}}\right)^{\beta_{i}}}{x}$.

This long-term average cost searches the minimal value $\phi_{i}^{*}=\phi_{i}\left(x_{i}^{*}\right)$, with $x_{i}^{*}$ denotes the optimal interval length. From equation (6), one obtains:

$x_{i}^{*}=\lambda_{i} \sqrt[\beta_{i}]{\frac{C_{i}^{p}}{C_{i}^{c}\left(\beta_{i}-1\right)}}$,

and the minimal long-term average cost:

$\phi_{i}^{*}=\phi_{i}\left(x_{i}^{*}\right)=\frac{C_{i}^{p} \beta_{i}}{x_{i}^{*}\left(\beta_{i}-1\right)}$.

This optimal interval length $x_{i}^{*}$ represents a nominal preventive maintenance frequency of component $i$ $(i=1, \ldots, N)$ and can be used to define tentative execution times which help to identify optimal maintenance occasions in which several components are performed.

\subsection{Step 2: Tentative planning}

\subsubsection{Defining the scheduling horizon}

In order to evaluate the performance of the dynamic grouping, a finite planning horizon is usually defined according to the current date, denoted $t_{\text {begin }}$, and the ending date $t_{\text {end }}$ that guarantees all components are preventively maintained at least one time in this horizon interval $\left[t_{\text {begin }}, t_{\text {end }}\right]$.

$\infty>t_{\text {end }}>\max _{i=1: N} t_{i^{1}}$,

where $t_{i}^{1}$ denotes the first tentative maintenance execution time of component $i(i=1, \ldots N)$ in this horizon.

$t_{i^{1}}=t_{\text {begin }}-t_{i}^{e}+x_{i}^{*}+D_{i}^{\sum}$

with $t_{i}^{e}$ is the operational time elapsed from the last preventive maintenance of component $i$ before $t$, and 
$D_{i}^{\sum}$ is the cumulative maintenance durations before the execution of component $i . D_{i}^{\sum}$ is added since the system is stopped during maintenance.

With this definition all components of the system are taken into account for the maintenance decision. To reduce the complexity time of grouping computation, we can choose:

$t_{\text {end }}=t_{j^{1}}+d_{j}$, with $t_{j^{1}}=\max _{i=1: N} t_{i^{1}}$.

\subsubsection{Tentative execution times}

It is assumed in (Wildeman, Dekker, \& Smit 1997) that each component is preventively maintained only one time in the planning horizon. In practice however, a system is composed of different components with different life time cycles. For example, engine oil has to be changed more frequently than driving belt on a heavy vehicle, (Bouvard, Artus, Berenguer, \& Cocquempot 2011). Herein, we consider components can be preventively maintained several times in the considered horizon planning.

Let $i^{j}$ be the $j$ th occurrence of maintenance activity $i$ (or component $i$ ) in the scheduling horizon, the tentative execution time of operation $i^{j}$, denoted $t_{i^{j}}$ $(j \geq 2)$, depends on the executed time $t_{i^{j-1}}^{*}$ of operation $i^{j-1}$, the cumulative maintenance durations $D_{i^{j-1}}^{\sum}$ from $t_{i^{j-1}}^{*}$, and the nominal periodicity $x_{i}^{*}$, see Figure 1.

$t_{i^{j}}=t_{i^{j-1}}^{*}+D_{i^{j-1}}^{\sum}+x_{i}^{*}$

$t_{i^{j-1}}^{*}=t_{G^{l}}$ : executed date of the group $G^{l}$.

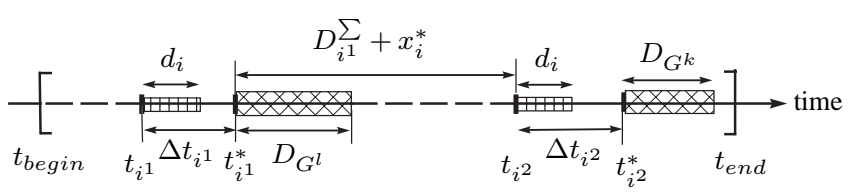

Figure 1: Illustration of dynamic grouping.

A remaining problem relying on how to identify $G^{l}$ will be investigated in the next section.

\subsubsection{Penalty cost calculation}

If the $j$ th $(j=1, \ldots)$ occurrence of maintenance activity $i$ is actually executed at time $t_{i^{j}}^{*}=t_{i^{j}}+\Delta t_{i^{j}}$ instead of the tentative execution time $t_{i^{j}}$, then the resulting additional cost is expressed by the penalty cost $h_{i}\left(\Delta t_{i^{j}}\right)$ which is written as, see (Cho \& Parlar 1991, Wildeman, Dekker, \& Smit 1997).

$h_{i}\left(\Delta t_{i^{j}}\right)=\Gamma_{i}\left(x_{i}^{*}+\Delta t_{i^{j}}\right)-\left(\Gamma_{i}\left(x_{i}^{*}\right)+\Delta t_{i^{j}} \cdot \phi_{i}^{*}\right)$,

with $\Delta t_{i^{j}}>-x_{i}^{*}$. By using Equation 5, we obtain:

$h_{i}\left(\Delta t_{i^{j}}\right)=M_{i}\left(x_{i}^{*}+\Delta t_{i^{j}}\right)-M_{i}\left(x_{i}^{*}\right)-\Delta t_{i^{j}} \cdot \phi_{i}^{*}$,
Another kind of penalty cost function can be found in (Bouvard, Artus, Berenguer, \& Cocquempot 2011).

From equations (4) and (8), we obtain finally:

$$
\begin{aligned}
h_{i}\left(\Delta t_{i^{j}}\right) & =C_{i}^{c} \cdot\left[\left(\frac{x_{i}^{*}+\Delta t_{i^{j}}}{\lambda_{i}}\right)^{\beta_{i}}-\left(\frac{x_{i}^{*}}{\lambda_{i}}\right)^{\beta_{i}}\right] \\
& -\Delta t_{i^{j}} \frac{C_{i}^{p} \beta_{i}}{x_{i}^{*}\left(\beta_{i}-1\right)} .
\end{aligned}
$$

The penalty costs will be used in the grouping optimization procedure to find all optimal groups with maximal cost savings in the scheduling horion.

\subsection{Step 3: Grouping optimization}

The main idea of this sept is to find all optimal maintenance occasions in order to minimize the expected maintenance cost in the considered scheduling horizon. In each maintenance occasion, several optimal maintenance operations can be alternatively performed. Here, we develop the algorithm introduced in (Wildeman, Dekker, \& Smit 1997, Wildeman \& Dekker 1997) by taking into account:

- duration of maintenance activities

- dependencies between occurrence dates of a maintenance activity

- grouping several occurrence of the same maintenance activity is forbidden.

\subsubsection{Mathematical formulation}

A maintenance occasion (or a group) is defined by three parameters: the execution date, the maintenance operations, and the duration. Two first parameters are optimally identified in order to minimize the expected maintenance cost. The later is the sum of the duration of all maintenance operations that are in the group.

Assume now the several deferent maintenance operations $i^{j}(i, j=1,2, \ldots)$ are performed in a group $G^{k}$ with $k \in \mathbb{N}$. Remember here that operations $i^{j}$ and $i^{l}$ $(j \neq l)$ are identical operations since they are respectively the $j$ th and the $l$ th occurrence of maintenance activity $i$. Let $H_{G^{k}}(t)$ be the group penalty cost function at time $t$. The optimal execution time of the group $t_{G^{k}}$ can be found when the $H_{G^{k}}($.$) searches its mini-$ mal value $H_{G^{k}}^{*}$. That is:

$H_{G^{k}}^{*}=H_{G^{k}}\left(t_{G^{k}}\right)=\min _{t}\left(\sum_{i^{j} \in G^{k}} h_{i}\left(t-t_{i^{j}}\right)\right)$

As mentioned above, the execution of a group of maintenance operations requires only one set-up cost, the group $G^{k}$ yields a cost reduction:

$U\left(G^{k}\right)=\left(\operatorname{card}\left(G^{k}\right)-1\right) \cdot S$. 
If the group set-up cost reduction $U\left(G^{k}\right)$ is larger or equal to the minimal group penalty cost $H_{G^{k}}^{*}, G^{k}$ is then called cost-effective (Wildeman, Dekker, \& Smit 1997), as consequence, the group savings $Q_{G^{k}}\left(t_{G}^{k}\right)$ are greater than or equal to zero, where:

$Q_{G^{k}}^{*}=U\left(G^{k}\right)-H_{G^{k}}^{*}$.

Based on the cost-effective groups, a grouping structure (or partition of all maintenance operations within the scheduling horizon) $S G^{l}$ (with $l=1,2, \ldots$ ) can be identified. The maximal cost savings $Q_{\Sigma}^{*}$ can be calculated as follows:

$Q_{\Sigma}^{*}=\max _{l=1,2, \ldots}\left\{\sum_{G^{k} \in S G^{l}} Q_{G^{k}}^{*}\right\}$.

In order to reduce the number of possible groups, it is shown in (Wildeman, Dekker, \& Smit 1997) that the optimal grouping structure, which can lead to the maximum total cost savings $Q_{\sum}^{*}$ in the scheduling horizon, groups maintenance operations whose tentative execution dates are consecutive.

Without loss generality, we renumber maintenance operations by increasing order according to their tentative execution times. A group will be defined based on several consecutive operations. In order to find cost-effective groups, for each maintenance operation $n(n=1,2, \ldots)$, we define interval $I_{n}$ in which the maintenance execution is cost-effective:

$I_{n}=\left[t_{n}+\Delta t_{n}^{-}, t_{n}+\Delta t_{n}^{+}\right]$,

with $t_{n}$ is the tentative execution time of the operation $n$, and $\Delta t_{n}^{-}, \Delta t_{n}^{+}$are respectively the smallest and the largest solution of the equation $h_{n}(\Delta t)-S=0$. This interval $I_{n}$ shows the maximum allowable shift (backward or forward) of the execution time of operation $n$.

We consider now a group $G^{k}=\{n, n+1, \ldots, k-$ $1, k\}$ with $1 \leq n \leq k$, it can be part of a grouping structure, so-called admissible group, if the following properties are verified:

- the intersection of intervals $I_{i, \forall i \in G^{k}}$ is not empty, $\bigcap_{i \in G^{k}} I_{i} \neq \emptyset$;

- the optimal execution time $t_{G^{k}}$ of $G^{k}$ is in $\bigcap_{i \in G^{k}} I_{i}$;

- the maintenance operations in $G^{k}$ must be different.

- the group $G^{k}$ must be irreducible, i.e. the group can not be divided into two or more subgroups that lead to a higher cost saving.
This procedure can provide the list of admissible groups which allow identifying the optimal grouping structure. Indeed, it is shown in (Wildeman, Dekker, $\&$ Smit 1997) that for the first $k$ operations an admissible group $G^{k}=\{l, \ldots, k\}$, with $1<l \leq k$, is the last optimal group (the last part of the optimal grouping structure) and the previous optimal one is an admissible group $G^{l-1}=\{, \ldots, l-1\}$ and so on. This is socalled the backtracking procedure.

\subsubsection{Occurrence maintenance operations problem}

To identify an admissible group $G^{k}$ that contains an operation $i^{j}(i=1,2, \ldots$ and $j>1)$, we have to calculate firstly the tentative execution $t_{i^{j}}$. As it is shown in Equation (11), $t_{i j}$ can be determined according to the optimal group $G^{l}$ that contains the operation $i^{j-1}$. However, the optimal group $G^{l}$ can be identified only when all admissible groups have been available. To this end, we consider the following theorem.

Theorem 1 If a recurrent operation $i^{j}$ is performed in an admissible group $G^{k}=\left(c, \ldots, i^{j}, \ldots, k\right)$, it previous occurrence (operation $i^{i-1}$ ) is then performed in an admissible group $G^{l}$ that is an optimal group of the first $(c-1)$ operations.

Proof. Based on the backtracking procedure, the following property can be easily verified:

Property 1 For two admissible groups $G^{e}=\{\ldots, e\}$ and $G^{p}=\{l, \ldots, p\}$ with $e<l \leq p$, if $G^{e}$ is an optimal group of the first $(l-1)$ operations and $G^{p}$ is an optimal group of the first $q(q \geq p)$ operations, $G^{e}$ is then also an optimal one of the first $q$ operations.

Using this property, it is clear that if $G^{k}$ is an optimal group in the scheduling horizon, $G^{l}$ is then an optimal one. This means that $i^{j-1}, i^{j}$ are performed in $G^{l}$ and $G^{k}$ respectively. Hence, the theorem is verified.

\subsection{Step 4: Update and decision}

Due to the previous step, we have an optimal grouping structure within the finite planning horizon $\left[t_{\text {begin }}, t_{\text {end }}\right]$. However, with time some new information (like maintenance resources constraints relying on for example management and/or new technology, opportunities, ...) by which this optimal grouping structure can be impacted may become available. To update the maintenance planning, a new optimal grouping structure within a new period must be identified. To this end, we simply go back to step 2. A new maintenance planning can be established without losing the optimality of the previous one thanks to the following important property.

Property 2 If an admissible groups $G^{l}=\{\ldots, l\}$ is an optimal group of the first $k(k>l)$ operations, it is then an optimal group of the first $p(p>k)$ operations.

This property can be easily proved according to the backtracking procedure. 
In this section, we will show how the preventive maintenance planning based on the developed dynamic grouping approach can be updated when occurring of an opportunity with restricted duration (e.g. the system is shutdown during a specific period for whatever reason) in which some preventive maintenance activities could be performed.

\subsection{Opportunities for preventive maintenance}

We assume that each opportunity $v(v=1,2, \ldots)$ is described by three parameters $t, T_{o p p}^{v}$, and $D_{o p p}^{v}$ :

- $t$ is the instant where the opportunity $v$ 's information is available

- $T_{o p p}^{v}$ is the beginning time of opportunity $v$

- $D_{o p p}^{v}$ represents the maximal duration of the opportunity.

The system is planned to be out of service within this opportunity duration. This means that if a maintenance operation $i(i=1,2, \ldots)$ is carried out in this interval, the unavailability $\operatorname{cost} c_{i}^{d}$ relying the production lost can be then removed. Therefore, we have to pay only a cost $S+c_{i}^{p}$.

\subsection{Grouping maintenance planning update}

After application of previous developed dynamic grouping approach, we have an optimal grouping maintenance planning within a finite scheduling horizon $\left[t_{\text {begin }}, t_{\text {end }}\right]$. Assume that at time $t\left(t_{\text {begin }}<t \leq\right.$ $\left.t_{\text {end }}\right)$ opportunity information is available, for example an opportunity will occur at $T_{o p p}\left(T_{o p p}>t\right)$ with limited duration $D_{\text {opp }}$. To find which maintenance operations should be performed in the opportunity, we have to reconstruct the grouping maintenance planning taking into account this opportunity. If at time $t$, a maintenance occasion (a group) is in process, the new grouping maintenance planning will be then applied after this maintenance occasion. The illustration of the online planning update process is presented in Figure 2.

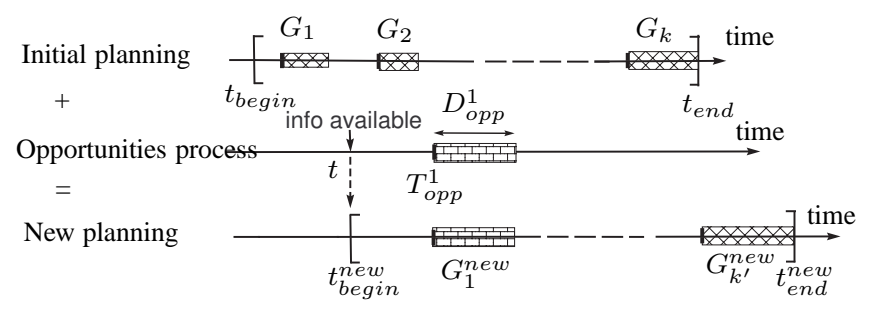

Figure 2: Illustration of grouping planning update with opportunity.

To establish a new grouping maintenance planning, we firstly go back to step 2 (i) to redefine the new

scheduling interval in order that all components are taken into account for the maintenance decision, and (ii) to determine the first tentative execution times of all maintenance operations within the new scheduling interval. We go next to step 3 to identify all optimal groups. To this end, we add a fictive maintenance operation, denoted operation opp, in which its tentative execution time is $T_{o p p}$ and its maintenance duration $d_{o p p}=0$. If the operation $о p p$ is in an admissible group, the execution time of the group is then $T_{\text {opp }}$.

Consider now a group $G^{p}=\{\ldots, o p p, \ldots, p\}$ with $1 \leq$ opp $\leq p$, it can be an admissible group if the following properties are verified:

- the intersection of intervals $I_{i, \forall i \in G^{p}}$ is not empty, $\bigcap_{i \in G^{p}} I_{i} \neq \emptyset$, and contains $T_{\text {opp }}, T_{\text {opp }} \in \bigcap_{i \in G^{p}} I_{i}$;

- the optimal execution time is $T_{o p p}$;

- the total duration of all operations in the group must be lower or equal to the opportunity duration, $\sum_{i \in G^{p}} d_{i} \leq D_{\text {opp }}$;

- the maintenance operations in $G^{p}$ must be different;

- the group $G^{p}$ must be irreducible, i.e. the group can not be divided into two or more subgroups that lead to a higher cost saving.

If $G^{p}$ is an admissible group, the corresponding cost savings is then:

$$
Q_{G^{p}}^{*}=U\left(G^{p}\right)-H_{G^{p}}\left(T_{o p p}\right)+\sum_{i \in G^{p}} d_{i} \cdot C^{d} .
$$

\section{NUMERICAL EXAMPLE}

The purpose of this section is to show how the proposed dynamic grouping approaches can be used in preventive maintenance optimization through a simple example of five series components system in which each component is preventively replaced after a fixed interval to be optimized. When a component fails, it is immediately maintained according to a minimal-repair policy. A failure repair restores the component involved into a state as good as before. We assume failure rate of a component $i(i=1, \ldots, 5)$ is described by a Weibull distribution with scale parameter $\lambda_{i}>0$, and shape parameter $\beta_{i}>1$.

Table 1 reports the random data for five components. For set-up cost and unavailability cost rate, we take $S=30$ and $C^{d}=20$.

The values of $C_{i}^{p}, x_{i}^{*}, \phi_{i}^{*}$ and $t_{i^{1}}$ calculated by substitution of the data input in Equations (2), (7), (8) and (9) respectively are given in Table 2. 
Table 1: Data of five components system.

\begin{tabular}{cccccc}
\hline Component $i$ & 1 & 2 & 3 & 4 & 5 \\
\hline$\lambda_{i}$ & 159 & 108 & 49 & 97 & 84 \\
$\beta_{i}$ & 1.7 & 1.7 & 1.25 & 1.75 & 1.5 \\
$c_{i}^{p}$ & 225 & 585 & 105 & 345 & 345 \\
$C_{i}^{c}$ & 182 & 172 & 90 & 50 & 76 \\
$d_{i}$ & 5 & 3 & 4 & 2 & 8 \\
$t_{i}^{e}$ & 92 & 120 & 149 & 32 & 110 \\
\hline
\end{tabular}

Table 2: Values of $x_{i}^{*}, \phi_{i}^{*}$, and $t_{i^{1}}$.

\begin{tabular}{cccccc}
\hline Component $i$ & 1 & 2 & 3 & 4 & 5 \\
\hline$C_{i}^{p}$ & 355 & 675 & 215 & 415 & 535 \\
$x_{i}^{*}$ & 142.3 & 249.7 & 179.2 & 273.8 & 230.1 \\
$\phi_{i}^{*}$ & 4 & 6.6 & 6 & 3.5 & 7 \\
$t_{i^{1}}$ & 54.3 & 151.7 & 30.2 & 270.8 & 129.1 \\
\hline
\end{tabular}

\subsection{Grouping maintenance planning}

To define the scheduling horizon, we used Equation (10): $t_{\text {end }}=t_{4^{1}}+d_{4}=272.8$ units of time.

Assume that all components are individually maintained, a maintenance planning based on the nominal maintenance periodicity $x_{i}^{*}(i=1, \ldots, 5)$ is shown in Figure 3 . According to this individual based planning components 1 and 3 are preventively maintained two times and the others are preventively performed only one time in the scheduling interval $\left[0, t_{\text {end }}\right]$. The total maintenance duration in which the system is stopped is $D^{\sum}=2 . d^{1}+d^{2}+2 . d^{3}+d^{4}+d^{5}=31$ units of time. The total expected maintenance costs for this interval period is:

$T C 1=\sum_{i=1}^{5}\left(t_{\text {end }}-D^{\Sigma}\right) \cdot \phi_{i}^{*}=6537.77$

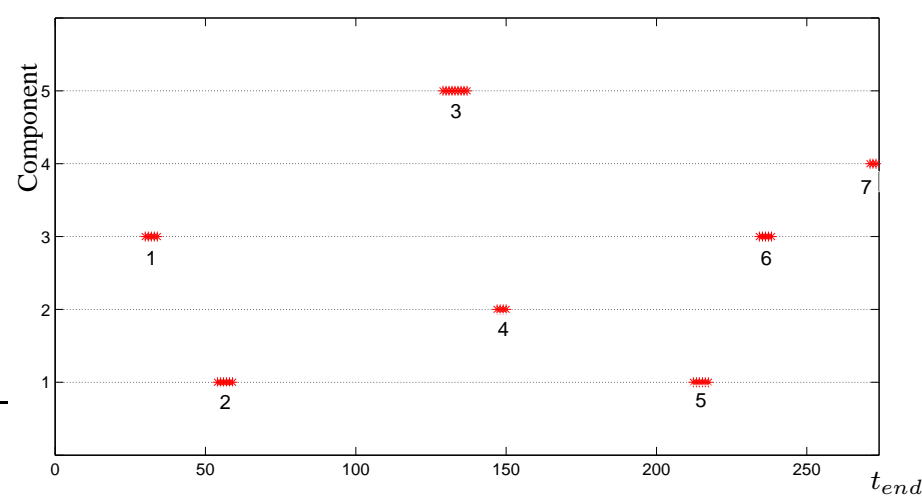

Figure 3: Individual based maintenance planning.

We consider now the grouping maintenance strategy. To identify all optimal groups, we applied the proposed dynamic grouping approach presented in Section 3. Within the scheduling interval, 7 maintenance operations are firstly named by increasing order numbers according to their tentative execution dates. Table 3 reports all admissible groups that can help to identify the optimal groups. Indeed, according to the backtracking, the optimal groups are $G^{7}=\{5,6,7\}$, $G^{4}=\{3,4\}$ and $G^{2}=\{1,2\}$. As consequence, the total cost savings are $Q_{\sum}^{*}=45.70+29.59+28.61=$ 103.90. When compared to the individual based planning in the same interval horizon, the grouping planning provides a cost saving of $1.59 \%$. Figure 44 sketches the grouping maintenance planning illustrated by blue color in the considered scheduling horizon.

Table 3: List of admissible groups.

\begin{tabular}{ccccc}
\hline$N^{o}$ & Ad. group & Optimal date & Duration & Savings \\
\hline$G^{1}$ & $\{1\}$ & 30.2 & 4 & 0 \\
$G^{2}$ & $\{1,2\}$ & 47 & 9 & 28.61 \\
$G^{3}$ & $\{3\}$ & 129.1 & 8 & 0 \\
$G^{4}$ & $\{3,4\}$ & 134.5 & 11 & 29.59 \\
$G^{5}$ & $\{5\}$ & 212.3 & 5 & 0 \\
$G^{6}$ & $\{5,6\}$ & 215.1 & 9 & 24.94 \\
$G^{7}$ & $\{5,6,7\}$ & 221.9 & 11 & 45.7 \\
\hline
\end{tabular}

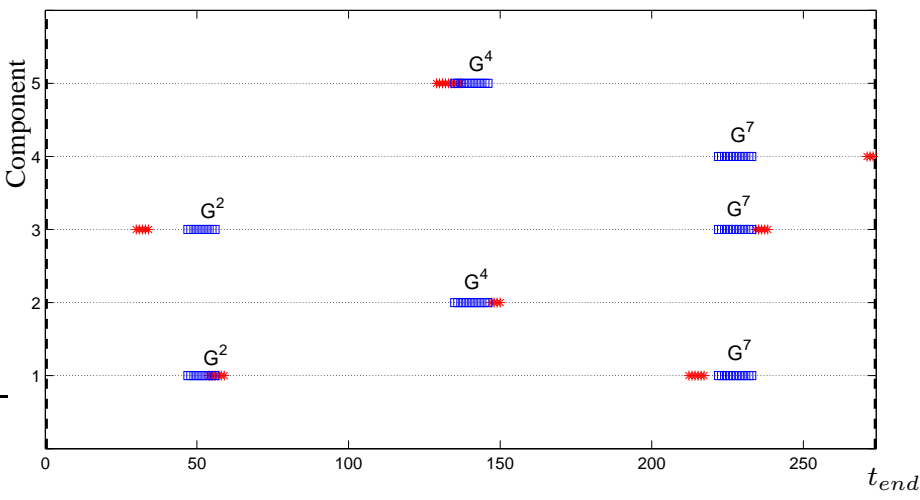

Figure 4: Grouping maintenance planning.

\subsection{Online update of grouping maintenance planning}

The grouping planning obtained above can be used as an optimal maintenance planning within the considered scheduling interval $[0,272.8]$ if the information by which the maintenance planning may be impacted remains unchanged. Assume now that at time $t=60$ the information concerning an opportunity is available: the opportunity will take place at $T_{o p p}=100$ within a limited duration $D_{o p p}=15$. The current grouping maintenance planning may be therefore no longer an optimal one. To update the maintenance planning, we have to go back to step 2. And the new scheduling interval obtained is $[60,287.8]$.

\subsubsection{Maintenance planning update without taking into account opportunity}

To show the advantages of our second proposed algorithm (presented in Section 4), we consider firstly the case where the opportunity is not taken into account. According to the first algorithm presented in 


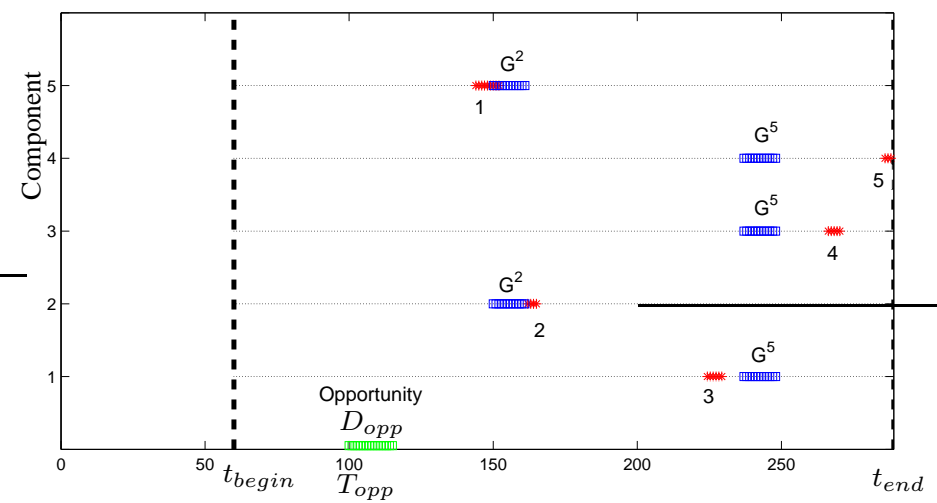

Figure 5: Updated grouping maintenance planning without taking into account opportunity.

Section 3, maintenance activities within the scheduling interval $[60,287.8]$ are named by increasing order numbers according to their tentative execution dates and the new grouping maintenance planning is established, see Figure 5. The opportunity illustrated by green color is not used to preventive maintenance. The total cost savings, with respect to the expected maintenance cost when all maintenance operations are never grouped together within the same considered scheduling horizon, are $Q_{\sum}^{*}=75.29$.

\subsubsection{Maintenance planning update with opportunity}

In order to take into account this opportunity, we applied the second proposed algorithm. A fictive maintenance operation named operation number 1 according to its tentative execution time $t_{1^{1}}=T_{\text {opp }}=100$ is added. Remember that by adding this fictive operation, the operation order names are here not the same as those above. The duration of the fictive operation $d^{1}$ is set to zero $\left(d^{1}=0\right)$.

By applying the groups' finding procedure presented in Section 4.2, all admissible groups are reported in Table 4. According to the backtracking, the optimal groups are $G^{6}=\{4,5,6\}$ and $G^{3}=\{1,2,3\}$. The total cost savings, with respect to the expected maintenance cost when all maintenance operations are separately carried out within the same considered scheduling horizon, are $Q_{\sum}^{*}=45.70+229.26=$ 274.96 .

Table 4: List of admissible groups in presence of opportunity.

\begin{tabular}{ccccc}
\hline$N^{o}$ & Ad. group & Optimal date & Duration & Savings \\
\hline$G^{1}$ & $\{1\}$ & 100 & 0 & 0 \\
$G^{2}$ & $\{1,2\}$ & 100 & 8 & 153.45 \\
$G^{3}$ & $\{1,2,3\}$ & 100 & 11 & 229.26 \\
$G^{4}$ & $\{4\}$ & 213.3 & 5 & 0 \\
$G^{5}$ & $\{4,5\}$ & 219.1 & 9 & 24.94 \\
$G^{6}$ & $\{4,5,6\}$ & 225.9 & 11 & 45.70 \\
\hline
\end{tabular}

When compared to the grouping maintenance planning above, this new one taking into account opportunity provides better results in term of maintenance cost.

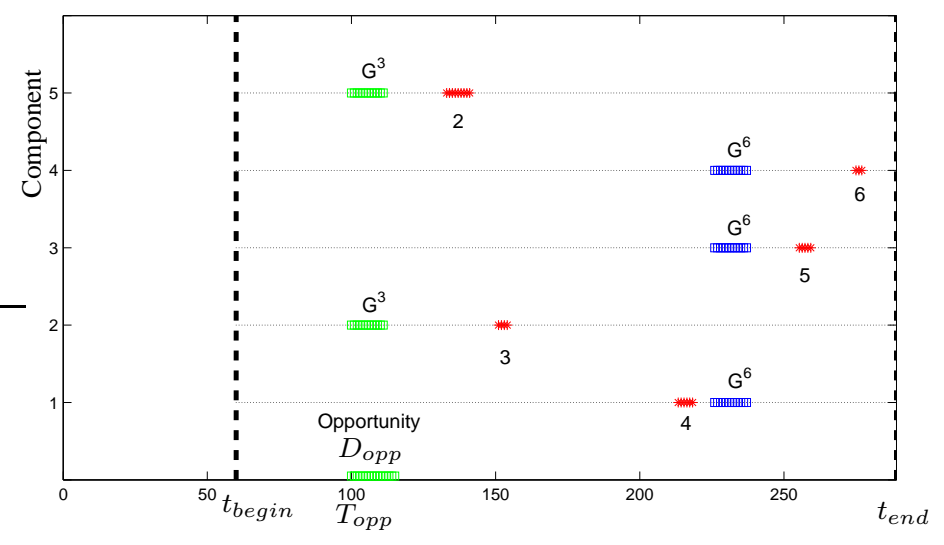

Figure 6: Updated grouping maintenance planning in presence of opportunity.

Figure 5 shows the new grouping maintenance planning in presence of opportunity in the considered scheduling horizon. The opportunity illustrated by green color is here profited to execute the preventive maintenance of components 2 and 5 .

\section{CONCLUSIONS}

In this work, firstly the rolling horizon approach introduced recently is extended to find the grouping maintenance planning of multi-component systems by taking into account the preventive maintenance durations and the occurrences of maintenance operations in the scheduling horizon. Minimal repair and block preventive replacement policy is used. Moreover, the paper proposed secondly a new algorithm in order to take into account some opportunities with limited durations, which should be profited to execute some maintenance activities, in the grouping optimization procedure. From a practical point of view, this can be therefore a powerful tool to update online the grouping maintenance planning when court term information is available.

Our future research work will focus on the development of the proposed approaches with availability/maintenance constraints of systems with inter-component dependencies. Furthermore, condition based maintenance will be also developed in this dynamic grouping maintenance framework.

\section{ACKNOWLEDGMENTS}

This work has been supported and partly financed by the European project MoDe - Maintenance on Demand (http://fp7-mode.eu) funded from the European Community's 7th Framework Program (Project SCP8-GA-2009-233890 - FP7 Sustainable Surface Transport).

\section{REFERENCES}

Bouvard, A., S. Artus, C. Berenguer, \& V. Cocquempot (2011). Condition-based dynamic maintenance operations planning 
and grouping. application to comercial heavy vehicules. $R e$ liability Engineering and System Safety.

Cho, D. \& M. Parlar (1991). A survey of maintenance models fo rmulti-unit systems. European Journal of Operational Research 51.

Dekker, R. (1995). Integrating optimisation, priority setting, planning and combining of maintenance activities. European Journal of Operational Research 82.

Dekker, R. \& E. Smeitink (1991). Preventive maintenance at opportunities with restricted duration. Serie Research Memoranda.

Nicolai, R. \& R. Dekker (2007). A review of multi-component maintenance models. In T. A. . J. E. Vinnem (Ed.), Risk, Reliability and Societal Safety - Proc.ESREL 2007, 25-27 June 2007, Stavanger, Norway, pp. 289-296. Taylor \& Francis.

Radouane, L., C. Alaa, \& A. Djamil (2009). Opportunistic policy for optimal preventive maintenance of a multi-component system in continuous operating units. Computers \& Chemical Engineering 9(9), 1499-1510.

Wildeman, R. \& R. Dekker (1997). Dynamic influences in multicomponent maintenance. Quality and reliability engineering international 13.

Wildeman, R., R. Dekker, \& A. Smit (1997). A dynamic policy for grouping maintenance activities. European Journal of Operational Research 99. 